\title{
ON THE METHOD OF PSEUDOPOTENTIAL FOR SCHRÖDINGER EQUATION \\ WITH NONLOCAL BOUNDARY \\ CONDITIONS
}

YURIY VALENTINOVICH ZASORIN

Received 7 July 2001

For stationary Schrödinger equation in $\mathbb{R}^{n}$ with the finite potential the singular pseudopotential is constructed in the form allowing us to find wave functions. The method does not require the knowledge of the explicit form of a potential and assumes only knowledge of the scattering amplitude for fixed level of energy.

\section{Introduction}

The stationary Schrödinger equation

$$
\left(\vec{\nabla} \cdot \vec{\nabla}+\lambda_{0}^{2}\right) \Psi(\vec{r})-q(\vec{r}, \Psi, \vec{\nabla} \Psi)=0 \quad \text { in } \mathbb{R}^{n}
$$

with the finite potential $q$ and nonlocal boundary condition (some spectral characteristics can be considered, scattering amplitude, for example) appears in certain problems of theoretical, nuclear, and quantum physics, using semiclassical Hartry-Fock-Slatter model (cf. [1]), in inverse problem of scattering theory (see $[4,5])$, and so forth. The method of pseudopotential, often used for study of these problems, is contained in replacement of potential $q$ by pseudopotential $\hat{q}$ (which does not depend explicitly on $\Psi, \vec{\nabla} \Psi$ ), of such form that the solution $\hat{\Psi}$ of the reduced problem coincides with $\Psi$ in exterior to effective area of the potential $q$. In contrast to methods of pseudopotential used up to now (cf. $[1,4,5]$ ), the new method, proposed in this article, does not require the knowledge of the explicit form of the potential $q$.

\section{Basic notation and preliminary results}

Let $\mathbb{R}^{n}$ be the Euclidean space of vectors $x=\left\{x_{1}, \ldots, x_{n}\right\}$ and let $r=|x|$ be the Euclidean length of the vector $x \in \mathbb{R}^{n}$. Let $\theta=x / r$ be a point of the unit

Copyright (C) 2001 Hindawi Publishing Corporation Abstract and Applied Analysis 6:6 (2001) 329-338 2000 Mathematics Subject Classification: 35J10, 35Q40, 35Q51, 35Q55

URL: http://aaa.hindawi.com/volume-6/S1085337501000689.html 
sphere $\omega=\{|x|=1\}$ and let $|\omega|$ be the area of $\omega$. Let $D=\left\{D_{1}, \ldots, D_{n}\right\}$, where $D_{j}=\partial / \partial x_{j} ; \Delta=D \cdot D$ is the Laplace operator in $\mathbb{R}^{n}$.

As usual (cf. [6]) we denote by $\mathscr{A}_{k}\left(\mathbb{R}^{n}\right), k=0,1, \ldots$, the space of degree $k$ homogeneous harmonic polynomials $Y_{k}(x)$ and by $\mathscr{A}_{k}(\omega)$ the space of their restrictions, $Y_{k}(\theta)=r^{-k} Y_{k}(x)$, to the unit sphere $\omega$. These polynomials $Y_{k}(x), Y_{k}(\theta)$ are called spherical harmonics of order $k$. Let $\mathscr{L}_{k}(x, y)$ be a zonal harmonic of order $k$ :

$$
\mathscr{L}_{k}(x, y)=\left(C_{k}^{v}(1)\right)^{-1}|x|^{k}|y|^{k} C_{k}^{v}\left(|x|^{-1}|y|^{-1}(x \cdot y)\right),
$$

where $C_{k}^{v}(z)$ is the Gegenbauer polynomial (see [3]), $v=(n-2) / 2$.

The following equalities hold:

$$
\begin{array}{r}
\mathscr{L}_{k}(x, y)=\mathscr{L}_{k}(y, x) ; \quad \mathscr{L}_{k}(\lambda x, y)=\mathscr{L}_{k}(x, \lambda y)=\lambda^{k \mathscr{L}_{k}(x, y) ;} \\
\Delta_{(x)} \mathscr{L}_{k}(x, y)=\Delta_{(y)} \mathscr{L}_{k}(x, y)=0 ; \\
\int_{\omega} Y_{m}\left(\theta^{\prime}\right) \mathscr{L}_{k}\left(\theta, \theta^{\prime}\right) d \omega\left(\theta^{\prime}\right)= \begin{cases}0 ; & m \neq k, \\
Y_{k}(\theta), & m=k .\end{cases}
\end{array}
$$

As usual (cf. [6]) $S\left(\mathbb{R}^{n}\right)$ is the Schwartz space of test rapidly decreasing functions $\varphi(x)$ and $Z\left(\mathbb{R}^{n}\right)$ is the space of Fourier images $(\mathscr{F} \varphi)(x)$ of functions $\varphi(x) \in C_{0}^{\infty}\left(\mathbb{R}^{n}\right) \subset S\left(\mathbb{R}^{n}\right)$. Let $S^{\prime}\left(\mathbb{R}^{n}\right)$ be the Schwartz space of tempered distributions dual to $S\left(\mathbb{R}^{n}\right)$ and $Z^{\prime}\left(\mathbb{R}^{n}\right)$ the space of analytic functionals dual to $Z\left(\mathbb{R}^{n}\right)$ and $E^{\prime}\left(\mathbb{R}^{n}\right) \subset S^{\prime}\left(\mathbb{R}^{n}\right)$ the space of compactly supported distributions dual to $C_{0}^{\infty}\left(\mathbb{R}^{n}\right)$.

LEMMA 2.1. For each distribution $T \in E^{\prime}\left(\mathbb{R}^{n}\right)$ and for each radial function $f(x) \equiv f_{0}(x) \in C_{0}^{\infty}\left(\mathbb{R}^{n}\right)$, it holds that

$$
\left\langle T(y) ; \mathscr{L}_{k}(x, y) f_{0}(|y|)\right\rangle \in \mathscr{A}_{k}\left(\mathbb{R}^{n}\right) .
$$

Proof. The proof follows immediately from relations (2.3) and (2.4).

In $S^{\prime}\left(\mathbb{R}^{n}\right)$ consider the following problem:

$$
\begin{gathered}
\left(\Delta+\lambda_{0}^{2}\right) u(x)=f(x), \quad x \in \mathbb{R}^{n} ; \\
\left(\frac{\partial}{\partial r}+i \lambda_{0}\right) u(x)=o\left(r^{(1-n) / 2}\right), \quad r=|x| \longrightarrow+\infty,
\end{gathered}
$$

where $\lambda_{0}=$ const $>0, i=\sqrt{-1}, u \in S^{\prime}\left(\mathbb{R}^{n}\right), f \in E^{\prime}\left(\mathbb{R}^{n}\right)$, moreover,

$$
\operatorname{supp}(f) \subset V_{0}=\left\{|x| \leq R_{0}\right\}, \quad R_{0}>0 .
$$

Problem (2.6) and (2.7) is well posed in $S^{\prime}\left(\mathbb{R}^{n}\right)$ (as well as in $Z^{\prime}\left(\mathbb{R}^{n}\right)$ ), its solution $u(x)$ has the form

$$
u(x)=\left\langle f(y), T_{0}(|x-y|)\right\rangle,
$$


where the radial distribution

$$
T_{0}(|x|)=-i 4^{-1}\left(\frac{\lambda_{0}}{2 \pi r}\right)^{v} H_{v}^{(1)}\left(\lambda_{0} r\right), \quad r=|x|, v=\frac{n-2}{2},
$$

is the fundamental solution of the Helmholtz equation

$$
\left(\Delta+\lambda_{0}^{2}\right) T_{0}(|x|)=\delta(x) .
$$

Here $H_{v}^{(1)}(z)=J_{v}(z)+i N_{v}(z)$ is the Hankel function (cf. [3]) and $\delta(\cdot)$ is the Dirac $\delta$-measure.

In the capacity of preliminary results we formulate the problem of construction of the singular multipolar pseudosource for problems (2.6) and (2.7). By the given source $f(x)$, construct a singular pseudosource $\hat{q}(x)$ with support concentrated at the point $\{x=0\}$, such that the problem

$$
\begin{gathered}
\left(\Delta+\lambda_{0}^{2}\right) \hat{w}(x)=\hat{q}(x), \quad x \in \mathbb{R}^{n}, \\
\left(\frac{\partial}{\partial r}+i \lambda_{0}\right) \hat{w}(x)=o\left(r^{(1-n) / 2}\right), \quad r=|x| \longrightarrow+\infty,
\end{gathered}
$$

is well posed simultaneously with problems (2.6) and (2.7) in some space of distributions, and, in addition, satisfies the identity

$$
\hat{w}(x) \equiv u(x), \quad|x|>R_{0} .
$$

The following assertion is valid.

LEMMA 2.2. The singular pseudosource $\hat{q}(x)$ and the corresponding solution $\hat{w}(x)$ of problems (2.11) and (2.12) can be represented in the form

$$
\begin{aligned}
\hat{q}(x) & =\sum_{k} \hat{q}_{k}(x)=\sum_{k}(-1)^{k} A_{k} Y_{k}(D) \delta(x), \\
\hat{w}(x) & =\sum_{k} \hat{w}_{k}(x)=\sum_{k}(-1)^{k} A_{k} Y_{k}(D) T_{0}(|x|) \\
& \equiv \sum_{k}(-i) C_{k} Y_{k}(x)\left(\frac{\lambda_{0}}{r}\right)^{\nu+k} H_{\nu+k}^{(1)}\left(\lambda_{0} r\right), \quad r=|x| ; \\
Y_{k}(x) & =\left\langle f(y) ; \mathscr{L}_{k}(x, y) j_{\nu+k}\left(\lambda_{0}|y|\right)\right\rangle ; \\
A_{k} & =\frac{\pi^{\nu+1}}{2^{k-1} \Gamma(v+k+1)}, \quad C_{k}=\frac{A_{k}}{2^{v+2} \pi^{v}}, \quad v=\frac{n-1}{2},
\end{aligned}
$$

where $j_{v}(z)=(2 / z)^{v} \Gamma(v+1) J_{v}(z)$ is the normalized Bessel function and $T_{0}(|x|)$ is the fundamental solution of Helmholtz equation (2.10), defined by equality (2.9). 
Remark 2.3. In the special case of $f(x) \in L^{2}\left(\mathbb{R}^{n}\right)$ Lemma 2.2 is proved in [7].

Remark 2.4. From [7,8], it follows that series (2.13) and (2.15) converge in the weak topology of $Z^{\prime}\left(\mathbb{R}^{n}\right)$, but do not converge in the weak topology of $S^{\prime}\left(\mathbb{R}^{n}\right)$; nevertheless their $N$ th partial sums $\hat{q}_{N}(x), \hat{w}_{N}(x)$ are distributions of $S^{\prime}\left(\mathbb{R}^{n}\right)$; moreover, the following equality is valid:

$$
\left\langle\hat{w}_{N} ; \varphi\right\rangle=\mathrm{v} \cdot \mathrm{p} \cdot \int_{\mathbb{R}^{n}} \hat{w}_{N}(x) \varphi(x) d x, \quad \forall \varphi \in S^{\prime}\left(\mathbb{R}^{n}\right),
$$

where v.p. is the Cauchy principal value

$$
\text { v.p. } \int_{\mathbb{R}^{n}} h(x) d x \triangleq \int_{0}^{+\infty} r^{n-1} d r \int_{\omega} h(r \theta) d \omega(\theta) .
$$

Proof of Lemma 2.2. First, note that (see [3]) $j_{v+k}\left(\lambda_{0} r\right) \in C^{\infty}\left(\mathbb{R}^{n}\right), r=|x|$, therefore (see Lemma 2.1) the right part of equality (2.16) is well defined.

Second, we prove the well-posedness of the right part of equality (2.15). Using the following property of Hankel functions (see [3]):

$$
\left(\frac{d}{z d z}\right)^{k}\left(z^{-v} H_{v}^{(1)}(z)\right)=(-1)^{k} z^{-v-k} H_{v+k}^{(1)}(z),
$$

and the fact (see [7]) that for each radial distribution (or function) $T_{0}(|x|$ ) and for each polynomial $Y_{k}(x) \in \mathscr{A}_{k}\left(\mathbb{R}^{n}\right)$ the following equality is valid:

$$
Y_{k}(x)\left(\frac{\partial}{r \partial r}\right)^{k} T_{0}(r)=Y_{k}(D) T_{0}(|x|), \quad r=|x|,
$$

we obtain

$$
\begin{aligned}
-i C_{k} Y_{k}(x)\left(\frac{\lambda_{0}}{r}\right)^{v+k} H_{v+k}^{(1)}\left(\lambda_{0} r\right) \\
=(-1)^{k+1} i C_{k} Y_{k}(x)\left(\frac{\partial}{r \partial r}\right)^{k}\left[\left(\frac{\lambda_{0}}{r}\right)^{v} H_{v}^{(1)}\left(\lambda_{0} r\right)\right] \\
=(-1)^{k+1} i C_{k} Y_{k}(D)\left[\left(\lambda_{0} r\right)^{v} H_{v}^{(1)}\left(\lambda_{0} r\right)\right] \\
=(-1)^{k} A_{k} Y_{k}(D) T_{0}(|x|)=\hat{w}_{k}(x),
\end{aligned}
$$

where the radial distribution $T_{0}$ and coefficients $C_{k}, A_{k}$ are defined by equalities (2.9) and (2.17), respectively. The well-posedness of formula (2.15) is proved.

Third, on the basis of equalities (2.10) and (2.15) we immediately obtain that the series (2.15) solves problem (2.11) with the singular pseudosource $\hat{q}(x)$, defined by equality (2.13). 
Finally, we prove the identity (2.12). From equality (2.9) it follows that $\operatorname{sing} \operatorname{supp}\left(T_{0}\right)=\{x=0\}$, hence, on the basis of equalities (2.7) and (2.8) we have that the distribution $u(x)$ is some real holomorphic function $H(x)$ at $\mathbb{R}^{n} \backslash V_{0}$. Next, using the properties of Hankel function (see [3, formulas (7.15.28), (7.15.29), (10.9.3), (10.9.5), (11.2.8)]) and formulas (2.1) and (2.9), we obtain

$$
\begin{gathered}
T_{0}(|x-y|)=\sum_{k}(-i) C_{k}\left(\frac{\lambda_{0}}{r}\right)^{v+k} \mathscr{L}_{k}(x ; y) j_{v+k}\left(\lambda_{0}|y|\right) H_{v+k}^{(1)}\left(\lambda_{0}|x|\right), \\
|y|<|x|, \quad v=\frac{n-1}{2},
\end{gathered}
$$

where $\mathscr{L}_{k}(\cdot ; \cdot)$ is a zonal harmonic, defined by equality (2.1) and coefficients $C_{k}$ are defined by formula (2.17). From here and from formulas (2.7) and (2.8), it follows that

$$
u(x)=\sum_{k}(-i) C_{k} Y_{k}(x)\left(\frac{\lambda_{0}}{r}\right)^{v+k} H_{v+k}^{(1)}\left(\lambda_{0} r\right), \quad r=|x|>R_{0},
$$

where harmonics $Y_{k}(x)$ are defined by equality (2.16).

Comparing equalities (2.15) and (2.24), we can see that these series are convergent simultaneously and uniformly to real holomorphic function $H(x)$ in $\mathbb{R}^{n} \backslash V_{0}$. Thus, identity (2.12) holds.

\section{Classical and quantum cases: pseudosource and pseudopotential}

At first, consider the classical (nonquantum) case when the wave function $u(x)$ does not create bound states, that is, the potential $q$ does not depend on $u, D u$ and actually is a source. But then we have a problem (2.6), (2.7), however the explicit form of the source $f(x)$ is unknown a priori (see $[4,5])$. Assume that only the scattering amplitude for the fixed level of energy $\lambda_{0}^{2}$ is known:

$$
\left\langle f(x) ; \exp \left[i \lambda_{0}(x \cdot \theta)\right]\right\rangle=F(\theta), \quad \theta \in \omega,
$$

or that the same

$$
\operatorname{Res}_{z=\lambda_{0}}\langle u(x) ; \exp [i z(x \cdot \theta)]\rangle=-\left(2 \lambda_{0}\right)^{-1} F(\theta), \quad \theta \in \omega,
$$

where $F(\theta)$ is a real holomorphic function on $\omega$.

Remark 3.1. Conditions (3.1) and (3.2) are equivalent, that is immediately proved by Fourier transform of (2.6).

Remark 3.2. On the other hand, condition (3.1) is insufficient in order to restore the distribution $f(x)$ (that can be easily verified in case of $n=1$ ). Moreover, for each $F(\theta)$ there are indefinite number of sources $f(x)$ and solutions $u(x)$ 
of problems (2.6) and (2.7), satisfying conditions (3.1) and (3.2), respectively. Nevertheless, construct the pseudosource $\hat{q}$ for problems (2.6) and (2.7), and (3.1) (or (2.6), (2.7), and (3.2)) satisfying the condition

$$
\left\langle\hat{q}(x) ; \exp \left[i \lambda_{0}(x \cdot \theta)\right]\right\rangle=F(\theta), \quad \theta \in \omega .
$$

Remark 3.3. It is necessary to note that the statement of the problem for the construction of the pseudosource $\hat{q}(x)$ is well posed by itself if condition (3.1) (or (3.2)) provides uniqueness of the restriction of all solutions $u(x)$ for problems (2.6), (2.7), and (3.1) (or (2.6), (2.7), and (3.2)) to the domain $\mathbb{R}^{n} \backslash V_{0}$. Later we will prove that this hypothesis is valid.

Construct the pseudosource $\hat{q}(x)$ in the form (2.13). On the basis of equality (3.1) we have

$$
\sum_{k} A_{k} Y_{k}\left(i \lambda_{0} \theta\right)=F(\theta)
$$

Represent the function $F(\theta)$ as a series

$$
F(\theta)=\sum_{k} \hat{Y}_{k}(\theta), \quad \hat{Y}_{k} \in \mathscr{A}_{k}(\omega) .
$$

Comparing formulas (3.4) and (3.5), we obtain

$$
Y_{k}(\theta)=\left(i \lambda_{0}\right)^{-k} A_{k}^{-1} \hat{Y}_{k}(\theta)=A_{k}^{-1} \hat{Y}_{k}\left(-i \lambda_{0}^{-1} \theta\right),
$$

or that the same

$$
Y_{k}(x)=A_{k}^{-1} \hat{Y}_{k}\left(-i \lambda_{0}^{-1} x\right)
$$

Consequently,

$$
\hat{q}(x)=\sum_{k} \hat{Y}_{k}\left(-i \lambda_{0}^{-1} D\right) \delta(x)
$$

and, on the basis of equality (2.15) we have

$$
\hat{w}(x)=\sum_{k}(4 i)^{-1} \hat{Y}_{k}(-i \theta)\left(\frac{\lambda_{0}}{2 \pi r}\right)^{v} H_{v+k}^{(1)}\left(\lambda_{0} r\right), \quad x=r \theta, v=\frac{n-2}{2} .
$$

We establish the relationship between the distribution $\hat{w}(x)$ (defined by equality (3.9)) and the solution $u(x)$ of problems (2.6), (2.7), and (3.1) (or (2.6), (2.7), and (3.2)), satisfying conditions (2.7), (3.1) and using the equality (see [7, 8])

$$
\exp \left[i \lambda_{0}(x \cdot \theta)\right]=\sum_{k}\left(i \lambda_{0}\right)^{k} A_{k} \mathscr{E}_{k}(x, \theta) j_{v+k}\left(\lambda_{0}|x|\right),
$$

where the coefficients $A_{k}$ are defined by equality (2.17), we have

$$
F(\theta)=\sum_{k}\left(i \lambda_{0}\right)^{k} A_{k}\left\langle f(x) ; \mathscr{L}_{k}(x, \theta) j_{v+k}\left(\lambda_{0}|x|\right)\right\rangle .
$$


From here and from equalities (3.5) and (2.16) it follows that

$$
\hat{Y}_{k}(\theta)=\left(i \lambda_{0}\right)^{k} A_{k}\left\langle f(x) ; \mathscr{L}_{k}(x, \theta) j_{v+k}\left(\lambda_{0}|x|\right)\right\rangle,
$$

or, denoting $x$ by $y$ and $\theta$ by $x$ :

$$
\hat{Y}_{k}(x)=\left(i \lambda_{0}\right)^{k} A_{k}\left\langle f(y) ; \mathscr{L}_{k}(x, y) j_{\nu+k}\left(\lambda_{0}|y|\right)\right\rangle .
$$

Comparing this equality with equalities (2.16) and (2.17) we obtain

$$
u(x)=\sum_{k}(4 i)^{-1} \hat{Y}_{k}(-i \theta)\left(\frac{\lambda_{0}}{2 \pi r}\right)^{v} H_{\nu+k}^{(1)}\left(\lambda_{0} r\right), \quad r=|x|>R_{0} .
$$

From (3.9), (3.14) it follows that all solutions $u(x)$ of problems (2.6), (2.7), and (3.1) (or (2.6), (2.7), and (3.2)) coincide in the domain $\mathbb{R}^{n} \backslash V_{0}$ among themselves and with the distributions $\hat{w}(x)$. Thus, the following assertion holds.

LEMMA 3.4. The pseudosource $\hat{q}(x)$ for problems (2.6), (2.7), and (3.1) (or (2.6), (2.7), and (3.2)) and the corresponding solution $\hat{w}(x)$ of problems (2.11), (2.12), and (3.3) can be represented by equalities (3.8), (3.9), and (3.5), respectively. Besides, in the domain $\mathbb{R}^{n} \backslash V_{0}$ each solution of problems (2.6), (2.7), and (3.1) (or (2.6), (2.7), and (3.2)) can be represented by equality (3.14).

Now consider the quantum case. The corresponding semiclassical HartryFock-Slatter model can be represented (see $[1,5]$ ) by the following problem:

$$
\begin{gathered}
\left(\Delta+\lambda_{0}^{2}\right) u(x)-q(x, u, D u)=0, \quad x \in \mathbb{R}^{n} ; \\
\left(\frac{\partial}{\partial r}+i \lambda_{0}\right) u(x)=o\left(r^{(1-n) / 2}\right), \quad r=|x| \longrightarrow+\infty,
\end{gathered}
$$

with condition (3.2).

Assume that the explicit form of the potential $q(\cdot, \cdot, \cdot)$ is unknown. Suppose that

$$
\operatorname{supp}(q(x, u(x), D u(x))) \subset V_{0}=\left\{|x| \leq R_{0}\right\}, \quad R_{0}>0,
$$

and, in addition

$$
q(\cdot, \cdot, \cdot) \in C_{0}^{\infty}\left(\mathbb{R}^{n} \times \mathbb{R} \times \mathbb{R}^{n}\right), \quad q(x, u(x), \vec{v}(x)) \in L_{\mathrm{loc}}^{1}\left(\mathbb{R}_{x}^{n}\right)
$$

for all $u, \vec{v}=\left\{v_{1}, \ldots, v_{n}\right\} \in L_{\text {loc }}^{1}\left(\mathbb{R}^{n}\right)$.

Remark 3.5. Further, we will intentionally ignore questions connected with the existence and uniqueness of solutions of problems (3.16), (3.17), (3.18), and (3.2), because they are completely investigated in [2, 4]. However, it is necessary to explain in what sense equation (3.16) in $S^{\prime}\left(\mathbb{R}^{n}\right)$ or $Z^{\prime}\left(\mathbb{R}^{n}\right)$ is being understood. 
Note that on the basis of the restrictions (3.17) it follows (see [2, 4]) that any solution $u(x)$ of problems (3.16), (3.17), (3.18) and $D u(x)$ are summable functions (i.e., regular distributions). But then we have that $q(x, u(x), D u(x)) \in$ $L_{\text {loc }}^{1}\left(\mathbb{R}^{n}\right)$ and it generates the regular distribution $f(x)=q(x, u(x), D u(x)) \in$ $S^{\prime}\left(\mathbb{R}^{n}\right)$ or $Z^{\prime}\left(\mathbb{R}^{n}\right)$.

Therefore, we will understand (3.16) in $S^{\prime}\left(\mathbb{R}^{n}\right)$ or $Z^{\prime}\left(\mathbb{R}^{n}\right)$ as the following equality:

$$
\left\langle u,\left(\Delta+\lambda_{0}^{2}\right) \varphi\right\rangle-\langle q ; \varphi\rangle=0
$$

for all test functions $\varphi(x)$.

Assuming that problems (3.16), (3.17), (3.18), and (3.2) are solvable, we fix any solution $u_{0}(x)$ and denote

$$
f_{0}(x) \triangleq q\left(x, u_{0}(x), D u_{0}(x)\right)
$$

But then problems (3.16), (3.17), (3.18), and (3.2) are reduced to problems (2.6), (2.7), and (3.2) or (see Remark 3.1) — to problem (2.6), (2.7), and (3.1). Therefore, the following assertion is valid.

LEMMA 3.6. If problems (3.16), (3.17), (3.18), and (3.2) are solvable, then the pseudopotential $\hat{q}(x)$ and the corresponding solution $\hat{w}(x)$ of problems (2.11), (2.12), and (3.3) can be represented by equalities (3.8), (3.9), and (3.5), respectively. Any solution $u(x)$ of problems (3.16), (3.17), (3.18), and (3.2) can be represented in the domain $\mathbb{R}^{n} \backslash V_{0}$ by equality (3.14).

\section{Final result: classes of well-posedness of (2.11) and (3.3)}

We derive some simple but important estimates. Using equality (2.4) and other well-known properties of zonal harmonics (see [6]) we have

$$
\left|\hat{Y}_{k}(\theta)\right| \leq|\omega|^{-1} a_{k}\|F\|_{2, \omega}, \quad a_{k}=\frac{n+2 k-2}{2}\left(\begin{array}{c}
c n+k-1 \\
k-1
\end{array}\right),
$$

where $\|\cdot\|_{2, \omega}$ denotes the $L^{2}(\omega)$-norm.

On the other hand, using relations (2.7), (3.1) we have (see [7])

$$
\left|\hat{Y}_{k}(\theta)\right| \leq M \lambda_{0}^{2 N} R_{0}^{N+k} A_{k}
$$

for some constants $M, N \geq 0$; coefficients $A_{k}$ are defined by equality (2.17). 
Also we have (see [3])

$$
\left|H_{v}^{(1)}(z)\right| \leq 4 \pi^{-1}\left[2^{v} \Gamma(v+1) z^{-v}+z^{-1 / 2}\right] .
$$

Combining estimates (4.1), (4.2), and (4.3) with equality (3.9), we obtain

$$
\left|\hat{w}_{k}(x)\right| \leq b_{k}(r) \equiv 2|\omega|^{-1} a_{k} R_{0}^{k}\left[r^{1-n-k}+\frac{\lambda_{0}^{\nu+k-1 / 2}}{2^{v+k} \Gamma(v+k+1) r^{1 / 2}}\right] \cdot\|F\|_{2, \omega} .
$$

Estimate (4.4) directly leads to the following assertion.

LEMMA 4.1. The series (3.9) constructed the solution $\hat{w}(x)$ of problems (2.11) and (3.3) is uniformly convergent on each sphere $\omega_{r}=\{|x|=r\}, r>R_{0}$, and is majorized by a numerical series $\sum_{k} b_{k}(r)$, where the coefficients $b_{k}(r)$ are defined by the relation (4.4).

Finally, the following assertion is valid.

Theorem 4.2. (1) The Nth partial sums $\hat{q}_{N}, \hat{w}_{N}$ of series (3.8) and (3.9) are distributions of $S^{\prime}\left(\mathbb{R}^{n}\right)$, moreover, equalities (2.18) and (2.19) are valid.

(2) The series (3.8), (3.5), and (3.9) constructed the pseudopotential (pseudosource) $\hat{q}(x)$ and corresponding to it solution $\hat{w}(x)$ of problems (2.11) and (3.3) are convergent in weak topology of $Z^{\prime}\left(\mathbb{R}^{n}\right)$.

(3) The problems (2.11) and (3.3) are well posed in $Z^{\prime}\left(\mathbb{R}^{n}\right)$.

Proof. The assertion (1) of Theorem 4.2 follows directly from Remark 3.2.

Finally, it follows from [8, Theorem 3 and Proposition 12], it is sufficient to prove that the series (3.8) is the multiplicator in $Z^{\prime}\left(\mathbb{R}^{n}\right)$.

Let $(\mathscr{F} \hat{q})(y)$ be a Fourier image of $\hat{q}(x)$

$$
(\mathscr{F} \hat{q})(y)=\sum_{k} \lambda_{0}^{-k} \hat{Y}_{k}(y)
$$

On the basis of relations (4.2) and (2.17) it follows that series (4.5) converges in $\mathbb{R}^{n}$ to a certain function $\mathscr{F} \hat{q} \in C^{\infty}\left(\mathbb{R}^{n}\right)$. If $\varphi \in Z^{\prime}\left(\mathbb{R}^{n}\right)$, then $\mathscr{F} \varphi \in C_{0}^{\infty}\left(\mathbb{R}^{n}\right)$ and $\mathscr{F} \hat{q} \cdot \mathscr{F} \varphi \in C_{0}^{\infty}\left(\mathbb{R}^{n}\right)$, hence, $(\hat{q} * \varphi) \in Z\left(\mathbb{R}^{n}\right)$.

Combining Lemmas 3.4, 3.6, 4.1, and Theorem 4.2, we can make the main conclusion:

In the domain $\mathbb{R}^{n} \backslash V_{0}$, the structure of the wave function $u(x)$ does not depend on the choice of the potential $q$ and is completely defined by the scattering amplitude $F(\theta)$. 


\section{References}

[1] S. Albeverio, R. Høegh-Krohn, F. Gesztesy, and H. Holden, Some exactly solvable models in quantum mechanics and the low energy expansions, Proceedings of the Second International Conference on Operator Algebras, Ideals, and Their Applications in Theoretical Physics (Leipzig, 1983), Teubner-Texte Math., vol. 67, Teubner, Leipzig, 1984, pp. 12-28. MR 85i:81015. Zbl 0562.47009.

[2] F. A. Berezin and M. A. Shubin, Schrödinger Equation, Moscow State University, Moscow, 1993.

[3] A. Erdélyi, W. Magnus, F. Oberhettinger, and F. G. Tricomi, Higher Transcendental Functions. Vol. III, McGraw-Hill, New York, 1955. MR 16,586c. Zbl 0064.06302.

[4] R. G. Novikov and G. M. Khenkin, The $\bar{\partial}$-equation in the multidimensional inverse scattering problem, Uspekhi Mat. Nauk 42 (1987), no. 3(255), 93-152, 255. MR 89d:35043. Zbl 0674.35085.

[5] M. Rasolt and R. Taylor, Pseudopotential from the variable phase approach to scattering theory, J. Phys. F. 2 (1972), no. 2, 270-276.

[6] E. M. Stein and G. Weiss, Introduction to Fourier Analysis on Euclidean Spaces, Princeton Mathematical Series, Princeton University Press, New Jersey, 1971. MR 46\#4102. Zbl 0232.42007.

[7] Yu. V. Zasorin, Harmonic analysis in Schwartz spaces of distributions and some applications to nonclassical problems of mathematical physics, Siberian Math. J. 38 (1997), no. 6, 1112-1129, translated from Sibirsk. Mat. Zh. 38 (1997), no. 6, 1282-1299. MR 99c:46038. Zbl 0935.43004.

[8] - On the Newton principle for the Helmholtz equation, Comput. Math. Math. Phys. 37 (1997), no. 7, 804-816, translated from Zh. Vychisl. Mat. Mat. Fiz. 37 (1997), no. 7, 828-840. MR 98d:35038. Zbl 0940.35060.

Yuriy Valentinovich Zasorin: Research Institute of Mathematics, Voronezh State University, 1, University SQ., Voronezh, 394693, Russia

E-mail address: mathins@main.vsu.ru 


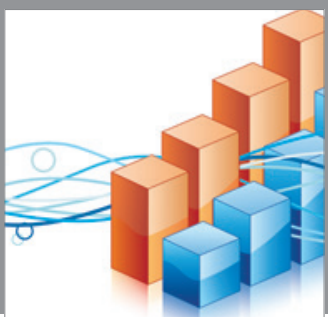

Advances in

Operations Research

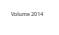

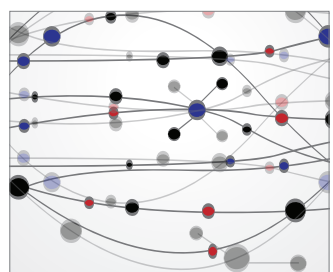

\section{The Scientific} World Journal
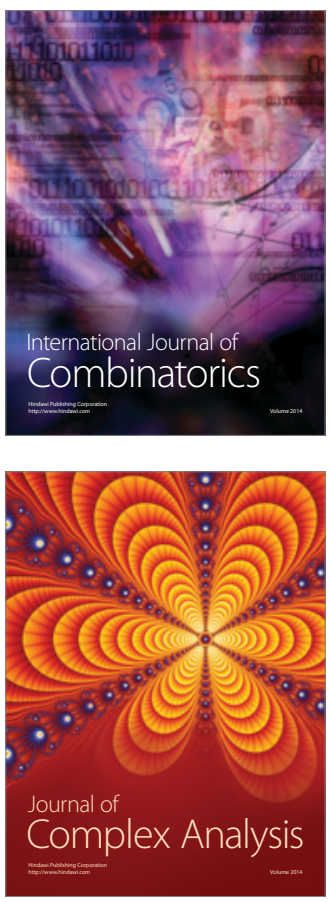

International Journal of

Mathematics and

Mathematical

Sciences
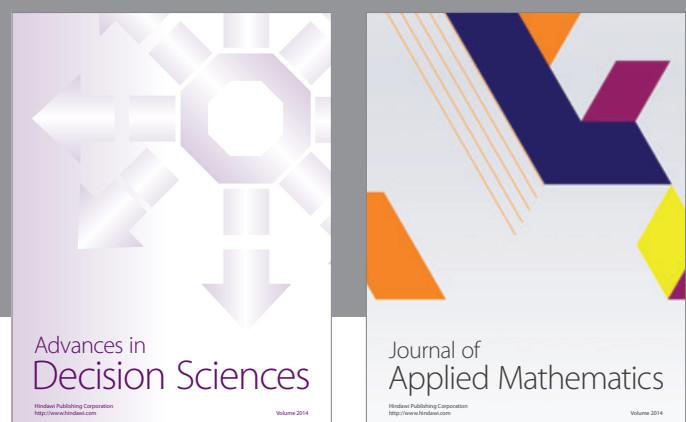

Journal of

Applied Mathematics
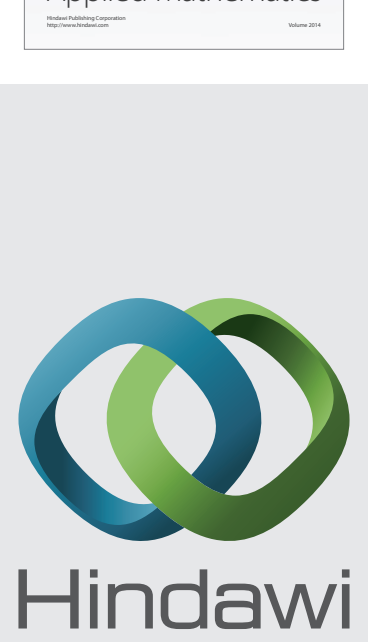

Submit your manuscripts at http://www.hindawi.com
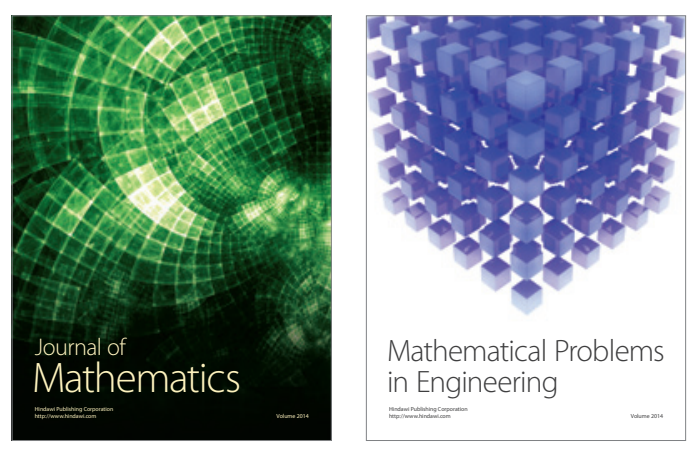

Mathematical Problems in Engineering
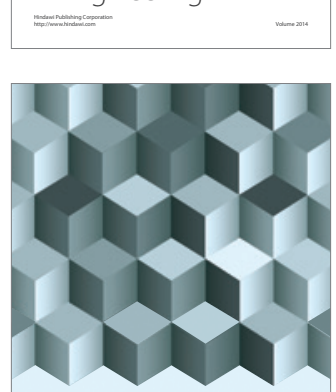

Journal of

Function Spaces
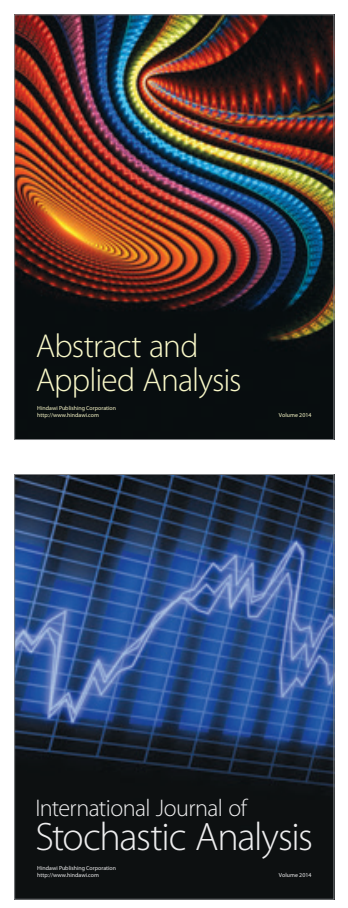

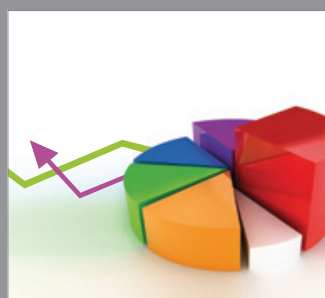

ournal of

Probability and Statistics

Promensencen
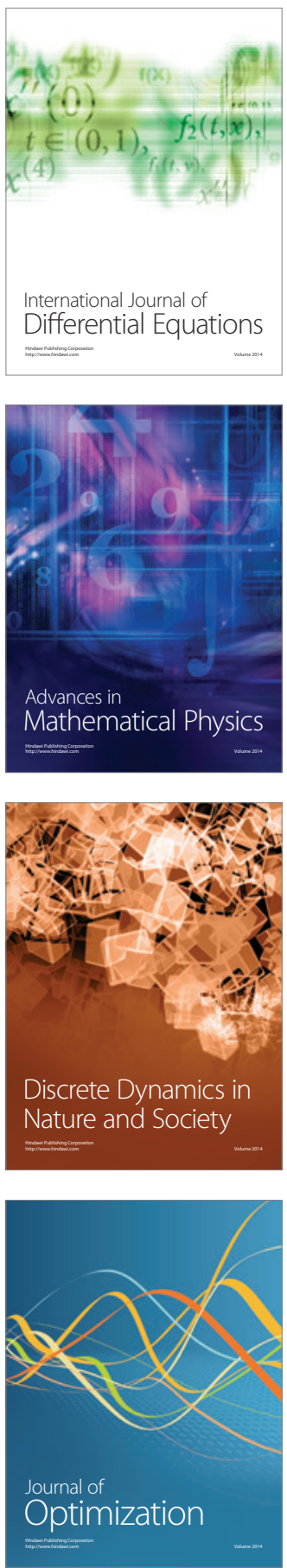